\title{
Poincare wave equations as Fourier transforms of Galilei wave equations
}

\author{
J. Gomis, A. Poch, and J. M. Pons \\ Department de Fïsica Teòrica, Universitat de Barcelona, Spain
}

(Received 17 April 1979; accepted for publication 2 November 1979)

The relationship between the Poincare and Galilei groups allows us to write the Poincare wave equations for arbitrary spin as a Fourier fransform of the Galilean ones. The relation between the Lagrangian formulation for both cases is also studied.

\section{INTRODUCTION}

It is well known that the Galilei algebra is a subalgebra of Poincaré algebra in one space dimension more. ${ }^{1}$ This fact allows us to relate relativistic Poincare and Galilean theories. An interesting point is that Galilei transformations in two space dimensions are contained in the usual Poincaré transformations. ${ }^{2}$ This enables us to present Poincaré spin zero wavefunctions as Fourier transforms of Galilean ones. In the same way it is possible to see the Klein-Gordon equation as the Fourier transform of the Schrödinger equation in one space dimension less.

On the other hand, due to the fact that the Poincare algebra is a subalgebra of the complex Galilei algebra in one space dimension more, ${ }^{3}$ it is possible to do a similar analysis as in the preceding case, i.e., the Schrödinger equation can be obtained as a Fourier transform of the Klein-Gordon equation. ${ }^{2}$

The aim of this paper is to extend the results above quoted to the arbitrary spin case and study the possible relations between the Lagrangian formulations of Poincaré and Galilei theories.

The organization of this paper is as follows: In Sec. 2 we give a summary of the results of Ref. 2 , in Sec. 3 we extend these results to the arbitrary spin case; in Sec. 4 we study some aspects of the Lagrangian formulation; Sec. 5 is devoted to conclusions.

\section{POINCARÉ AND GALILEI SPIN-0 WAVE EQUATIONS}

The light cone transformation

$t=\frac{1}{\sqrt{2}}\left(x^{0}-x^{3}\right), \quad x=x^{1}, \quad y=x^{2}$,

$s=\frac{1}{\sqrt{2}}\left(x^{0}+x^{3}\right)$,

induces $^{1}$ the $(2+1)$ Galilei algebra

$J=J_{3}, \quad: P_{1}=P_{1}, \quad \mathscr{P}_{2}=P_{2}$,

$G_{1}=\frac{1}{\sqrt{2}}\left(K_{1}+J_{2}\right), \quad G_{2}=\frac{1}{\sqrt{2}}\left(K_{2}-J_{1}\right)$,

$\mathscr{H}=\frac{1}{\sqrt{2}}\left(P_{0}-P_{3}\right), \quad M=\frac{1}{\sqrt{2}}\left(P_{0}+P_{3}\right)$,

where $P_{\mu}, \mathbf{J}$, and $\mathbf{K}$ are the generators of the $(3+1)$ Poincaré algebra.

If we take the linearized natural representation of the Poincaré group acting on $\left(x^{0}, x^{1}, x^{2}, x^{3}, 1\right)$ and subduce it to the Galilei subgroup generated by (2.2), we obtain, in the coordinates (2.1), the transformation

$t^{\prime}=t+b, \quad x_{1}^{\prime}=R x_{1}+v_{1} t+a_{1}$,

$\left[x_{1} \equiv\left(\begin{array}{l}x \\ y\end{array}\right)\right], \quad s^{\prime}=s+v_{1} x_{\perp}+\frac{1}{2} v_{\perp}^{2} t+\theta$,

which is the natural representation of the $(2+1)$ extended Galilei group.

Notice that the $s$ transformation is related to the phase that appears in the projective representations of the Galilei group. $^{4}$

Let us now relate the spin-0 Poincaré and Galilei wave functions by means of a Fourier transformation. A wavefunction $\psi\left(t, x_{1}, s\right)$ scalar under Poincaré group can be expressed as

$$
\psi\left(t, x_{1}, s\right)=\int d \eta e^{i \eta s} \phi_{\eta}\left(t, x_{\perp}\right),
$$

where $\phi_{\eta \prime}\left(t, x_{1}\right)$ is a scalar wavefunction under the $(2+1)$ extended Galilei group. Now we can write the Klein-Gordon equation as

$$
\left(\square+m^{2}\right) \psi\left(t, x_{\perp}, s\right)=\int d \eta e^{-i m}\left(S \phi_{n}\right)\left(t, x_{\downarrow}\right),
$$

where $\left(S \phi_{\eta}\right)$ is the Schrödinger equation for a $(2+1)$ Galilean particle of mass $\eta$.

On the other hand, the imaginary coordinate transformation ${ }^{2}$

$x^{0}=i z, \quad x^{1}=x, \quad x^{2}=y, \quad x^{3}=\gamma t, \quad \gamma$ arbitrary,

induces the $(2+1)$ Poincaré algebra

$$
\begin{aligned}
& \mathscr{Y}=J_{3}, \quad K_{1}=-i J_{2}, \quad K_{2}=i J_{1}, \\
& \mathscr{P}_{1}=P_{1}, \quad \mathscr{P}_{2}=P_{2}, \quad \mathscr{H}=-i P_{3},
\end{aligned}
$$

where $\mathbf{P}, \mathbf{J}$ are the generators of translations and rotations of the $(3+1)$ Galilei group.

As in the preceeding case, if we take the linearized natural representation of the extended Galilei group acting on $(x, y, z, t, s, 1)$ and subduce it to the Poincaré subgroup generated by (2.7), we can obtain, using (2.6), the natural representaiton of the $(2+1)$ Poincaré group.

A wavefunction $\phi(t, \mathbf{x})$ scalar under Galilei group can be written, using (2.6), as

$$
\phi\left(x^{0}, x^{1}, x^{2}, x^{3}\right)=\int d \lambda e^{-i \lambda x^{3}} \psi_{\lambda}\left(x^{0}, x^{1}, x^{2}\right),
$$

where $\psi_{i}\left(x^{0}, x^{1}, x^{2}\right)$ is a scalar wavefunction under the $(2+1)$ Poincare group. In the same way, the Schrödinger 
equation can be written as

$(S \phi)\left(x^{0}, x^{1}, x^{2}, x^{3}\right)=\int d \lambda e^{-i \lambda x^{3}}(\mathrm{~K}-\mathrm{G}) \psi_{\lambda}\left(x^{0}, x^{1}, x^{2}\right)$,

where $(\mathrm{K}-\mathrm{G}) \psi_{\lambda}$ is the Klein-Gordon equation in $(2+1)$ dimensions with mass $M^{2}=-2 m \gamma \lambda$.

Let us remark that the invariance of the Klein-Gordon equation implies, by (2.5), the invariance of the Schrödinger equation. Due to (2.9), the inverse is also true.

\section{POINCARE AND GALILEI SPIN S WAVE EQUATIONS}

We shall first concentrate ourselves in the Dirac equation for a spin $\frac{1}{2}$ particle of mass $m$. This equation is invariant under the $\mathscr{D} \equiv D^{(1 / 2,0) \otimes(0,1 / 2)}$ representation of $\operatorname{SL}(2, C)$ with generators

$$
\mathbf{J}=\frac{1}{2}\left(\begin{array}{cc}
\overline{\boldsymbol{\sigma}} & 0 \\
0 & \overline{\boldsymbol{\sigma}}
\end{array}\right), \quad \overline{\mathbf{K}}=\frac{i}{2}\left(\begin{array}{cc}
\overline{\boldsymbol{\sigma}} & 0 \\
0 & \overline{\boldsymbol{\sigma}}
\end{array}\right) .
$$

If this representation is subduced using (2.2), to the $(2+1)$ Galilei algebra, we obtain an equivalent representation of that of Levy-Leblond ${ }^{4}$ for spin $\frac{1}{2}$.

This equivalence can be expressed by a matrix $A$, whose explicit form is given by

$$
A=\left(\begin{array}{cccc}
1 & 0 & 0 & 0 \\
0 & 0 & 0 & -i \\
0 & 0 & \frac{1}{\sqrt{2}} & 0 \\
0 & \frac{-i}{\sqrt{2}} & 0 & 0
\end{array}\right) .
$$

Using this matrix, we obtain

$\tilde{J}=A J A^{-1}=\frac{1}{2}\left(\begin{array}{cc}\sigma_{3} & 0 \\ 0 & \sigma_{3}\end{array}\right)$,

$\tilde{G}=A G_{i} A^{-1}=\frac{1}{2}\left(\begin{array}{ll}0 & 0 \\ \sigma_{i} & 0\end{array}\right), \quad i=1,2$,

which is the usual representation of the Galilei algebra.

The matrix $A$ acts on Dirac spinors: $\psi_{\mathrm{D}}^{A}=A \psi_{\mathrm{D}}$. Now we can generalize (2.4), writing

$$
\psi_{\mathrm{D}}^{A}\left(t, x_{1}, s\right)=\int d \eta e^{-i \eta s} \phi_{\eta \mathrm{LL}}\left(t, x_{\perp}\right),
$$

where $\phi_{\eta \mathrm{LL}}$ is a Galilean wavefunction for a spin $\frac{1}{2}$ particle of mass $\eta$, and now we can write the Dirac equation for $\psi_{\mathrm{D}}^{A}$ as

$$
\begin{aligned}
A\left(\gamma_{\mu} P^{\mu}-m\right) A^{-1} \psi_{\mathrm{D}}^{A}\left(t, x_{1}, s\right) \\
\quad=\int d \eta e^{-i \eta s} G_{\eta} \phi_{\eta \mathrm{LL}}\left(t, x_{\perp}\right),
\end{aligned}
$$

where

$$
G_{\eta} \phi_{\eta \mathrm{LL}}=\left(\begin{array}{cc}
-i \sigma_{\perp} P_{1}-m & 2 \eta \\
E & i \sigma_{\perp} P_{\perp}-m
\end{array}\right)\left(\begin{array}{l}
\varphi_{\eta} \\
\chi_{\eta}
\end{array}\right),
$$

is the Levy-Leblond equation in $(2+1)$ dimensions for a particle of mass $\eta$ and spin $\frac{1}{2}$. Therefore, the Dirac equation is the Fourier transform of Levy-Leblond equations.

Let us search the transformations properties of the Ga- lilean spinor $\phi_{\eta \mathrm{LL}}$ from those of the corresponding Dirac spinor.

Under the Lorentz group, the Dirac spinor transforms as

$$
\psi_{\mathrm{D}}^{\prime}\left(x^{\prime}\right)=\mathscr{D}(\Lambda) \psi_{\mathrm{D}}(x) .
$$

If we restrict ourselves to transformations belonging to the $(2+1)$ homogeneous Galilei group, $A=A_{\mathrm{G}}$, the new Dirac spinor $\psi_{\mathrm{D}}^{4}$ transforms as

$$
\psi_{\mathrm{D}}^{\prime A}\left(t^{\prime}, x_{1}^{\prime}, s^{\prime}\right)=\Delta\left(\Lambda_{\mathrm{G}}\right) \psi_{\mathrm{D}}^{A}\left(t, x_{1}, s\right),
$$

where $\Delta\left(\Lambda_{\mathrm{G}}\right)=A \mathscr{D}\left(\Lambda_{\mathrm{G}}\right) A^{-1}$ is the $(2+1)$ Galilei subduced representation generated by (3.3).

Using (3.4), (3.7) is given by

$$
\begin{aligned}
& \int d \eta e^{-i \eta s^{\prime}} \phi_{\eta \mathrm{LL}}^{\prime}\left(t^{\prime} x_{\perp}^{\prime}\right) \\
& \quad=\Delta\left(\Lambda_{\mathrm{G}}\right) \int d \eta e^{-i \eta s} \phi_{\eta \mathrm{LL}}\left(t, x_{\perp}\right),
\end{aligned}
$$

and from (2.3) we have

$\phi_{\eta \mathrm{LL}}^{\prime}\left(t^{\prime}, \boldsymbol{x}_{\perp}^{\prime}\right)=e^{i f} \boldsymbol{\Delta}\left(\Lambda_{G}\right) \phi_{\eta \mathrm{LL}}\left(t, x_{\perp}\right)$,

where $f=\left[v_{1} x_{1}+\frac{1}{2} v_{\perp}^{2} t\right] \eta$ is the known phase which appears in the projective representations of the Galilei group. ${ }^{4}$

Now let us note that the operator (3.6) can be put in a more standard form. In order to eliminate the $m$-dependence we can define a new transformation on the spinors

$$
\left(\begin{array}{l}
\hat{\varphi}_{\eta} \\
\hat{\chi}_{\eta}
\end{array}\right)=e^{i\left(m^{2} / 2 \eta\right) t}\left(\begin{array}{cc}
I & 0 \\
-\frac{m}{2 \eta} & I
\end{array}\right)\left(\begin{array}{l}
\varphi_{\eta} \\
\chi_{\eta}
\end{array}\right),
$$

that induces a transformation of the operator (3.6), which becomes

$$
\left(\begin{array}{cc}
-i \sigma_{\perp} P_{\perp} & 2 \eta \\
E & i \sigma_{\perp} P_{\perp}
\end{array}\right)
$$

with a further change (3.9) takes the form

$$
\begin{gathered}
\left(\begin{array}{cc}
1 & 0 \\
0 & -i
\end{array}\right)\left(\begin{array}{cc}
-i \sigma_{\perp} P_{\perp} & 2 \eta \\
E & i \sigma_{\perp} P_{\perp}
\end{array}\right)\left(\begin{array}{ll}
i & 0 \\
0 & 1
\end{array}\right) \\
=\left(\begin{array}{cc}
\sigma_{\perp} P_{\perp} & 2 \eta \\
E & \sigma_{\perp} P_{\perp}
\end{array}\right) .
\end{gathered}
$$

Thus we have obtained the well-known classical form of the Levy-Leblond operator.

It is important to observe that while the operator of (3.9) is invariant under the $(2+1)$ Galilei group due to the invariance of the Dirac operator and the relation (3.5), the Levy-Leblond operator (3.10) is not an invariant one..$^{5,6}$

We can generalize this result to an arbitrary spin case. Let us begin with the Bargmann-Wigner ${ }^{7}$ procedure. The Bargmann-Wigner equations are

$$
\left(I \otimes \cdots \otimes D_{(i)}^{D} \otimes \cdots \otimes I\right) \psi_{\mathrm{BW}}\left(x^{\mu}\right)=0, \quad i=1 \cdots 2 s,
$$

where $D$ is the Dirac operator and $\psi_{\mathrm{Bw}}$ is a symetric multispinor of rank $2 s$. This equation are invariant under the $\mathscr{D} \otimes \cdots \otimes \mathscr{D}$ representation of $\operatorname{SL}(2, C)$. If we subduce this representation, using $(2.2)$, to the $(2+1)$ Galilei group, we obtain: 


$$
\Delta \otimes \cdots \otimes \Delta .
$$

The matrix $A$ acts on symmetric multispinors $\psi_{\mathrm{BW}}^{A}=A \otimes$ $\cdots \otimes A \psi_{\mathrm{Bw}}$, and analogoulsy to (3.4) we can write

$$
\psi_{\mathrm{BW}}^{A}\left(t, x_{1}, s\right)=\int d \eta e^{-i \eta s} \phi_{\mathrm{BW} \eta}\left(t, x_{\perp}\right)
$$

where $\phi_{\mathrm{BW} \eta}$ is a Galilean symmetric multispinor of rank $2 s$. As in spin $\frac{1}{2}$ case the BW equations for $\psi_{\mathrm{BW}}^{A}$ give

$$
\begin{gathered}
A \otimes \cdots \otimes A(I \otimes \cdots \otimes \underset{(i)}{D} \otimes \cdots \otimes I) A^{-1} \otimes \cdots \otimes A^{-1} \psi_{\mathrm{BW}}^{A}\left(t, x_{1}, s\right) \\
=\int d \eta e^{-i \eta s}\left(I \otimes \cdots \otimes G_{\eta}^{G_{\eta}} \otimes \cdots \otimes I\right) \phi_{\mathrm{BW} \eta}\left(t, x_{1}\right) \\
i=1 \cdots 2 s,
\end{gathered}
$$

where $\left(I \otimes \cdots \otimes G_{\eta} \otimes \cdots \otimes I\right) \phi_{\mathrm{Bw} \eta}\left(t, x_{1}\right)(i=1 \cdots 2 s)$ are the BW Galilean equations in $(2+1)$ dimensions for a particle of spin $s$ and mass $\eta$. Therefore, the $\mathbf{B W}$ equations are the Fourier transform of BW Galilean equations.

If we used another description for a spin $s$ particle, we can obtain simialar results. For example, if we use the reformulate $(6 s+1)$ Hurley theory ${ }^{8}$ as a modified BW set, ${ }^{9}$ i.e.,

$$
(\Gamma \otimes \cdots \otimes \underset{(i)}{D} \otimes \cdots \otimes \Gamma) \psi_{\mathrm{BW}}\left(x^{\mu}\right)=0, \quad i=1 \cdots 2 s,
$$

where $D$ is the Dirac operator and $\Gamma$ is the projector $\Gamma=\left(\begin{array}{ll}I & 0 \\ 0 & 0\end{array}\right) . \psi_{\mathrm{BW}}$ is a symmetric multispinor of rank $2 s$. Due to the fact that $\Gamma$ commutes with the $\mathscr{D}$ representation, of SL (2.C), these equations are invariant under the tensor product of the $\mathscr{D}$ representation.

If we subduce this representation using (2.2) we obtain the Galilei representation (3.12). As in the usual BW case (3.11), the equations (3.15) for $\psi_{\mathrm{BW}}^{4}$ can be written as

$$
\begin{array}{r}
A \otimes \cdots \otimes A(\Gamma \otimes \cdots \oplus \underset{(i)}{D} \otimes \cdots \otimes \Gamma) A^{-1} \otimes \cdots \otimes A^{-1} \psi_{\mathrm{BW}}^{A}\left(t, x_{1}, s\right) \\
=\int d \eta e^{-i \eta s}\left(P_{+} \otimes \cdots \otimes G_{(i)} \otimes \cdots \otimes P_{+}\right) \phi_{\mathrm{BW} \eta}\left(t, x_{1}\right) \\
i=1 \cdots 2 s
\end{array}
$$

where $\left(P_{+} \otimes \cdots \otimes G_{\eta} \otimes \cdots \otimes P_{+}\right) \phi_{\mathrm{BW}_{\eta}}$ is a Galilean equation in $(2+1)$ dimensions for a particle of spin $s$ and mass $\eta$ in the $(6 s+1)$ theory. $P_{+}$is a projector:

$$
P_{+}=A \Gamma A^{-1}=\left(\begin{array}{cccc}
1 & & & \\
& 0 & & \\
& & 0 & \\
& & & 1
\end{array}\right) \text {. }
$$

Equation $\left(P_{+} \otimes \cdots \otimes G_{\eta} \otimes \cdots \otimes P_{+}\right) \phi_{\mathrm{BW}_{\eta}}=0$ is an invariant Galilean equation owing to the fact that

$$
\Delta^{\dagger} P_{+} \Delta=P_{+} .
$$

Therefore, the Pincare $(6 s+1)$ theory is the Fourier transform of the Galilei $(6 s+1)$ theory.

Summing up, we can say that the possible Poincare multispinor wave equations can be written as Fourier transforms of the Galilean ones. On the other hand, we can span a symmetric multispinor in a basis of $\mathrm{SO}(3)$ tensors. Using this fact it is easy to see that the Poincare tensorial wave equa- tions can be written as Fourier transforms of Galilei tensorial wave equations.

We want to remark, that in this section we have only studied the Poincaré wavefunctions as Fourier transforms of the Galilei ones, a related analysis can be performed in the inverse situation.

\section{POINCARÉ AND GALILEI LAGRANGIANS}

The results of the preceeding sections suggest that it must exist a relation between the Poincare and Galilei Lagrangians. This is due firstly to the fact that Poincaré wavefunctions are a Fourier transform of the Galilei ones and secondly that the Lagrangians are bilinear functions of the fields.

Let us begin this analysis with the scalar case. The Lagrangian for the Klein-Gordon fields is given by

$$
\mathscr{L}_{\mathrm{KG}}\left(x^{0}, \mathbf{x}\right)=m^{2} \psi^{*} \psi+\mathbf{J}_{\mu} \psi^{*} \mathbf{J}^{\mu} \psi,
$$

if we use the coordinate transformation (2.1), (4.1) can be written as

$$
\begin{aligned}
\mathscr{L}_{\mathrm{KG}}\left(t, x_{1}, s\right)= & -m^{2} \psi^{*} \psi+\partial_{s} \psi^{*} \partial_{t} \psi \\
& +\partial_{t} \psi^{*} \partial_{s} \psi-\partial_{i} \psi^{*} \partial_{i} \psi
\end{aligned}
$$

Now, using (2.4), we have

$$
\begin{aligned}
\int \mathscr{L}_{\mathrm{KG}}\left(t, x_{\perp}, s\right) d s= & \int d \eta\left[-m^{2} \phi_{\eta}^{*} \phi_{\eta}-i \eta \partial_{t}\left(\phi_{\eta}^{*} \phi_{\eta}\right)\right. \\
& \left.+2 i \eta \phi_{\eta}^{*} \partial_{t} \phi_{\eta}-\partial_{i} \phi_{\eta}^{*} \partial_{i} \phi_{\eta}\right]
\end{aligned}
$$

Dimensional reasons suggest to redefine the Galilean fields

$$
\hat{\phi}_{\eta}\left(t, x_{1}\right)=(2 m \eta)^{1 / 2} \phi_{\eta}\left(t, x_{\perp}\right) \text {. }
$$

Now, (4.3) becomes

$$
\int \mathscr{L}_{\mathrm{KG}}\left(t, x_{\perp}, s\right) d s=\frac{1}{m} \int d \eta \mathscr{L}_{\mathrm{Sch} \eta}\left(t, x_{\perp}\right),
$$

where $\mathscr{L}_{\mathrm{Sch} \eta}$ is the usual Schrödinger Lagrangian with an additive term which is physically interpreted as an energy shift. Moreover we can write

$$
W_{\mathrm{KG}}=\int d \eta W_{\mathrm{Sch} \eta},
$$

being $W_{\mathrm{KG}}$ the action for the Klein-Gordon field, and $W_{\mathrm{S} c h \eta}$ the action for the Schrödinger field with mass $\eta$ in $(2+1)$ dimensions.

We want to remark that the relation (4.6) between the actions is not given by a Fourier transform. This means that if we make a variation $\delta W_{\mathrm{KG}}=0$ it does not imply $\delta W_{\mathrm{Sch} \eta}=0$, therefore we can not take (4.6) as a starting point in order to relate Poincaré and Galilean theories.

Now let us consider the relativistic Lagrangian for a spin- $\frac{1}{2}$ particle

$$
\mathscr{L}_{\mathrm{D}}\left(x^{0}, \mathbf{x}\right)=\psi^{\dagger} \gamma^{0}\left(\gamma_{\mu} P^{\mu}-m\right) \psi .
$$

If we write (4.7) in terms of the new Dirac spinor $\psi^{A}$, we have

$$
\mathscr{L}_{\mathrm{D}}\left(x^{0}, \mathbf{x}\right)=\psi^{A \dagger} A^{+-1} \gamma^{0}\left(\gamma_{\mu} P^{\mu}-m\right) A^{-1} \psi^{A},
$$

but under the coordinate transformation (2.1), (4.8) becomes

$$
\mathscr{L}_{\mathrm{D}}\left(t, x_{1}, s\right)
$$


$=\sqrt{2} \psi^{A}+\left(\begin{array}{cc}E & i \sigma_{\perp} P_{1}-m \\ -i \sigma_{\perp} P_{\perp}-m & 2 M\end{array}\right) \psi^{A}$,

being $E \equiv i \partial / \partial t$ and $M \equiv i \partial / \partial s$.

Using (3.4) and (4.9), we have

$$
\begin{aligned}
& \int \mathscr{L}_{\mathrm{D}}\left(t, x_{1}, s\right) d s \\
& \quad=\sqrt{2} \int d \eta \phi_{\eta}^{+}\left(\begin{array}{cc}
E & i \sigma_{1} P_{\perp}-m \\
-i \sigma_{1} P^{1}-m & 2 \eta
\end{array}\right) \phi_{\eta} .
\end{aligned}
$$

In order to cancel the $m$-dependence in the rhs of (4.10) and also to recover the dimensionality of the Levy-Leblond field we define

$\hat{\phi}_{\eta}\left(t, x_{\perp}\right)=\sqrt{m} e^{i\left(m^{x} / 2 \eta\right) t}\left(\begin{array}{cc}I & 0 \\ -\frac{m}{2 \eta} & I\end{array}\right) \phi_{\eta}\left(t, x_{\perp}\right)$.

Therefore, we have found

$\int \mathscr{L}_{\mathrm{D}}\left(t, x_{\perp}, s\right) d s=(\sqrt{2} / m) \int d \eta \mathscr{L}_{L\llcorner\eta}\left(t, x_{\perp}\right)$,

where $\mathscr{L}_{\mathrm{LL}, \eta}$ is the well-known Levy-Leblond Lagrangian for a particle of spin $\frac{1}{2}$.

In the same way as in the spin zero case, we also have

$$
W_{\mathrm{D}}=\int d \eta W_{\mathrm{LL} \eta},
$$

being $W_{\mathrm{D}}$ and $W_{\mathrm{LL} \eta}$, the actions for the Dirac and LevyLeblond fields, respectively. The remarks we have done for the spin- 0 case can serve here unaltered.

\section{CONCLUSIONS}

Due to the relation between the Poincaré and Galilei groups, the Poincaré and Galilei transformations are seen to be contained one each other in one space dimension more.
This fact allows us to write the Poincare wavefunctions as Fourier transforms of Galilean ones, and also to find the transformation properties of the Galilei wavefunctions under the $(2+1)$ Galilei group from the transformation properties of Poincaré wavefunctions under the $(3+1)$ Poincaré group. In particular it is easy to see the projective character of the representations of the $(2+1)$ dimensional Galilei group. Taking into account these last properties we can write the Poincaré wave equations for arbitrary spin as Fourier transforms of the Galilean ones, so the Dirac equation can be seen as the Fourier transform of the Levy-Leblond equation. The relation between Poincaré and Galilei wavefunctions allows us to the relate the Lagrangians of the two theories.

The generalization to the higher spin cases, the relation between the energy-momentum tensor of the two theories and the possible introduction of external fields in this framework is under investigation.

'J. Kogut and D. Soper, Phys. Rev. D 1, 2901 (1970); E. Elizalde and J. Gomis, Nucl. Phys. B 122, 535 (1977); J. Gomis and J.M. Pons: Nuovo Cimento A 47, 166 (1978).

${ }^{2}$ J. Gomis and J.M. Pons, Phys. Lett. A 66, 463 (1978).

${ }^{3}$ E. Elizalde, J. Math. Phys. 19, 526 (1978); E. Elizalde and J. Gomis, J. Math. Phys. 19, 1790.(1978).

${ }^{4}$ J.M. Levy-Leblond: Commun. Math. Phys. 6, 286 (1967) J.M. Levy-Leblond, Galilei group and Galilean Invariance, Vol. II, Group Theory and Its Applications, edited by E.M. Loeble (Academic, New York, 1971), Chapter V.

${ }^{5}$ U.H. Niederer and L. O'Raifeataigh, Ann. Inst. Henri Poincaré A, XXVI, 313 (1977), no. 3.

'We want to point that $(3.9)$ can be trivialy generalized to $(3+1)$ dimensions. Therefore we can have Galilean invariant operator instead of the usual Levy-Leblond one.

${ }^{7}$ V. Bargmann and E.P. Wigner, Proc. Nat. Acad. Sci. U.S.A. 34, 211 (1948).

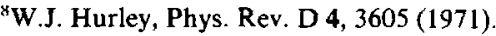

${ }^{9}$ J. Gomis and J.M. Pons, "The Bargmann-Wigner method and the Galilei and Poincaré $6 s+1$ theories", Universitat de Barcelona, preprint (1978). 